\title{
Evaluation of Potential Association between Nitric Oxide Synthase Gene Polymorphism and Psoriasis
}

K.M.Hussein ${ }^{1}$,S.H.Abdullah ${ }^{2}$, D.M.El habak ${ }^{1}$ and R.M.AbdelMawla ${ }^{1}$

${ }^{1}$ Dermatology, Venereology and Andrology Dept., Faculty of Medicine, Benha Univ., Benha, Egypt

${ }^{2}$ Biochemistry, Dept., Faculty of Medicine, Zagazig Univ., Egypt

E-Mail:rooka9999@gmail.com

\begin{abstract}
Attention has been focused on role of a genetically determined skin barrier dysfunction in the pathogenesis of psoriasis. A study has provided evidence for the correlation of psoriasis with a low expression of genes encoding filaggrin and loricrin in skin biopsies taken from both psoriatic plaques and perilesional skin and analyzed by real-time PCR method. Family of genes encoding late cornified envelope proteins (LCE proteins) which is located within on chromosome 1q21.3 complex has shown abnormalities in patients with psoriasis. The objectives of the present study were to evaluate the serum levels of nitric and to investigate single nucleotoide polymorphism in nitric oxide
\end{abstract}

Keywords: Nitric,Psoriasis, polymorphism,genes,history.

\section{Introduction}

Psoriasis is a chronic disease that is estimated to affect approximately $1.7 \%$ of population. Psoriasis is a multisystem inflammatory disease with predominantly skin and joint involvement [3]. It has a bimodal age of onset (16 to 22 and 57 to 60 years) and affects both sexes equally. Pathogenesis is multifactorial, involving dysregulated inflammation and genetic associations $[1,2$ and 3].

Psoriasis has a complex genetic background. Studies have shown that psoriasis represents a multifactorial disorder in which genetic factors account for about $70 \%$ of disease susceptibility, whereas environmental factors account for the remaining $30 \%$. Aditionally skin microbiota [4] may also contribute to the susceptibility to this immuno-inflammatory disease. Genome-wide association studies (GWAS) have led to the identification of numerous candidate genes that may play a role in the pathogenesis of psoriasis. These include genetic polymorphisms of genes involved in the interleukin (IL) 23, 12, 17 (IL-23, IL-12, IL-17) and nuclear factor $\mathrm{kB}(\mathrm{NF}-\mathrm{kB})$-dependent signaling pathway [ 2,3 and 4].

Psoriasis arises as a result of dysregulated interactions of the innate and adaptive immune system in the context of skin epithelium and connective tissue [5].

Nitric is a molecule that has attracted heightened interest due to its vast role in human health and biology $[6,7]$.

There are three identified NOS isoforms namely neuronal (nNOS or NOS1) and endothelial (eNOS or NOS3) and inducible iNOS) or NOS2 isoforms. The neuronal (nNOS) and endothelial(eNOS) are calciumdependent; whereas, the last one is inducible (iNOS or NOS2) and $\mathrm{Ca} 2+$ independent [8].

\section{Patients and methods}

Patients with psoriasis of both sexes within the age group (20-40 years old) versus otherwise apparently healthy subjects (number 10) within same age (20-40) of both sexes serving as controls were enrolled in the study. An informed written concent was explaind to then signed by each participant.
Diagnosis of psoriasis patients: The clinical diagnosis was done for each patient [9] and the severity of psoriasis for each patient was evaluated using psoriasis area severity index (PASI) score [10].

\section{Exclusion criteria}

Other dermatological diseases (such as vitiligo, Systemic lupus erythematosus, atopic dermatitis)

Pregnant and lactating females.

History of tuberculosis or any active infectious disease.

Systemic illness (eg. diabetes, hypertension, liver diseases renal disorders, rheumatoid disease or other arthropathy disorder)

History of drug intake within last six weeks (eg. Sildenafil, systemic corticosteroids, cyclosporine or methotrexate...etc)

Hypertensive patients (diastolic blood pressure more $90 \mathrm{mmHg}$ ).

Patients with BMI equal to more than 30 .

Patients with Psoriasis arthropathy.

\section{All patients was subjected to the followings}

Complete history taking.

Complete general examination with blood pressure measurment.

Detailed dermatological examination including type and sites of psoriasis and scoring of psoriasis by PASI score.

Complete blood picture (CBC) liver and renal functions tests were carried out.

Serum Nitric level Determination and Detection of gene polymorphism by PCR.

Statistical analysis

All data were collected, tabulated and statistically analyzed using SPSS 22.0 for windows (SPSS Inc., Chicago, IL, USA) \& MedCalc 13 for windows (MedCalc Software bvba, Ostend, Belgium). Data were tested for normal distribution using the Shapiro Walk test. Qualitative data were represented as frequencies and relative percentages. Chi square test $\left(\chi^{2}\right)$ and Fisher exact was used to calculate difference between qualitative variables as indicated. Quantitative data were expressed as mean $\pm \mathrm{SD}$ (Standard deviation) for 
parametric and median and range for non-parametric data. Independent $\mathrm{T}$ test and Mann Whitney test were used to calculate difference between quantitative variables in two groups for parametric and nonparametric variables respectively. One way ANOVA test supplied with post hoc (LSD) test was used to compare between more than two dependent groups of normally distributed variables. Pearson's and Spearman's correlation coefficient were used for correlating normal and non-parametric variables respectively.

3. Results

Patients with psoriasis (number 80) of both sexes within the age group (20-40 years old) versus otherwise apparently healthy subjects (number 30) within same age (20-40) of both sexes serving as controls were enrolled in the study. Demographic data in table (1).

Table (1) Demographic characteristics between the two groups.

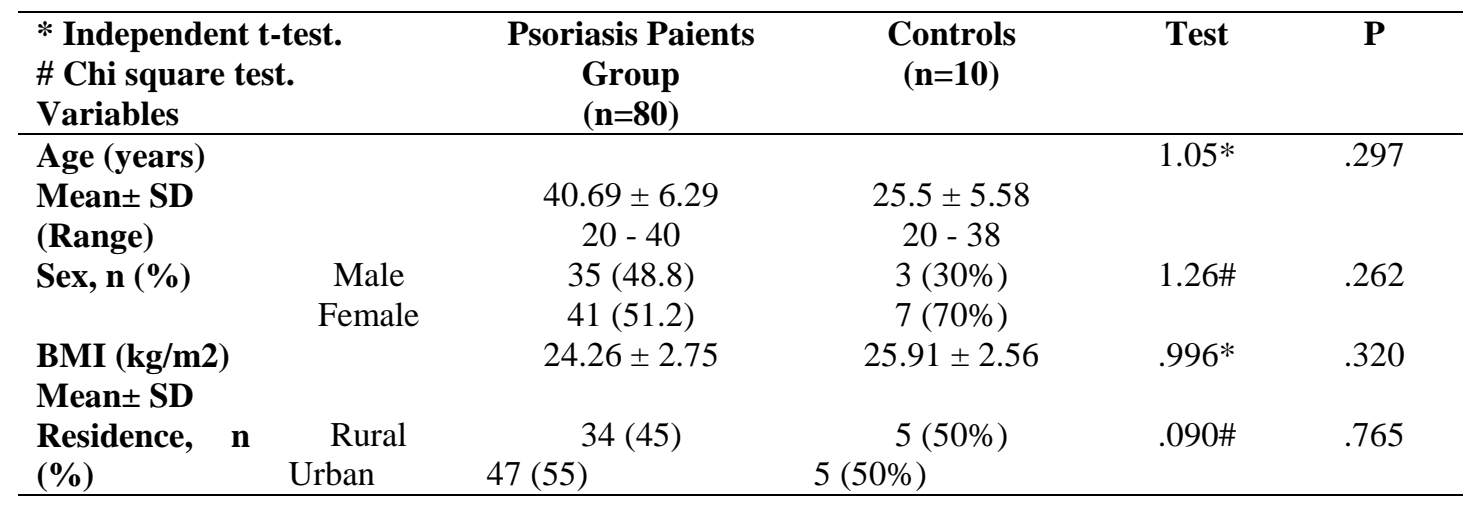

BMI: body mass index. SD: Standard deviation. Independent t-test.

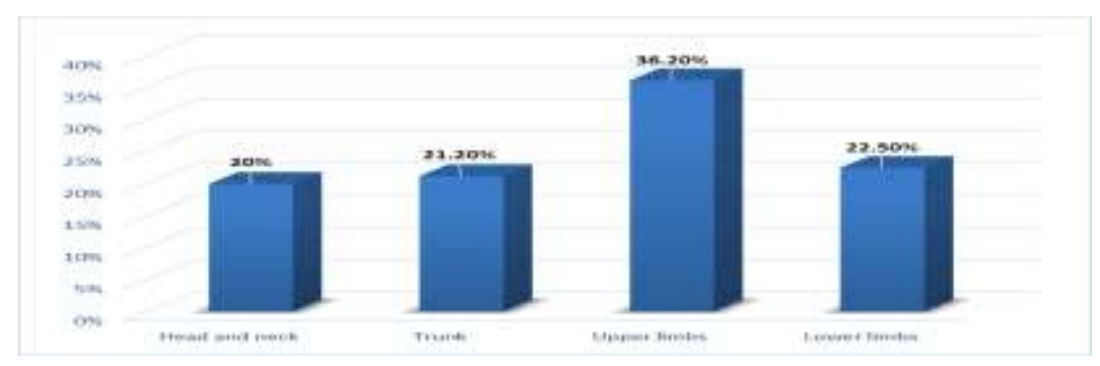

Fig (1) Affected site distributions among psoriasis patients.

Table (2) Socioeconomic status between the two groups.

\begin{tabular}{|c|c|c|c|c|c|c|}
\hline \multirow[t]{2}{*}{ Variables } & \multicolumn{2}{|c|}{$\begin{array}{c}\text { Cases } \\
(n=80)\end{array}$} & \multicolumn{2}{|c|}{$\begin{array}{c}\text { Controls } \\
(n=10)\end{array}$} & \multirow[t]{2}{*}{$\mathbf{x 2}$} & \multirow[t]{2}{*}{$\mathbf{P}$} \\
\hline & $\mathbf{N}$ & $\%$ & $\mathbf{N}$ & $\%$ & & \\
\hline Low & 20 & 25 & 1 & 10 & 1.81 & .614 \\
\hline Medium & 18 & 22.5 & 2 & 20 & & \\
\hline High & 23 & 28.7 & 3 & 30 & & \\
\hline Very high & 19 & 23.8 & 4 & 40 & & \\
\hline
\end{tabular}

Table (3) Psoriasis clinical characteristics of the study group.

\begin{tabular}{lcc}
\hline Variables & \multicolumn{2}{c}{ Psoriasis Patients(n=80) } \\
\hline PASI score & \\
Mean \pm SD & & $29.72 \pm 9.32$ \\
(Range) & & $15-45$ \\
Site, n (\%) & Head and neck & $15(20 \%)$ \\
& Trunk & $18(21.2 \%)$ \\
& Upper limbs & $28(36.2 \%)$ \\
& Lower limbs & $17(22.5 \%)$ \\
\hline
\end{tabular}

PASI, Psoriasis Area Severity Index. SD: standard deviation 2: Chi square test. Chi Square Test BMI and residence. 


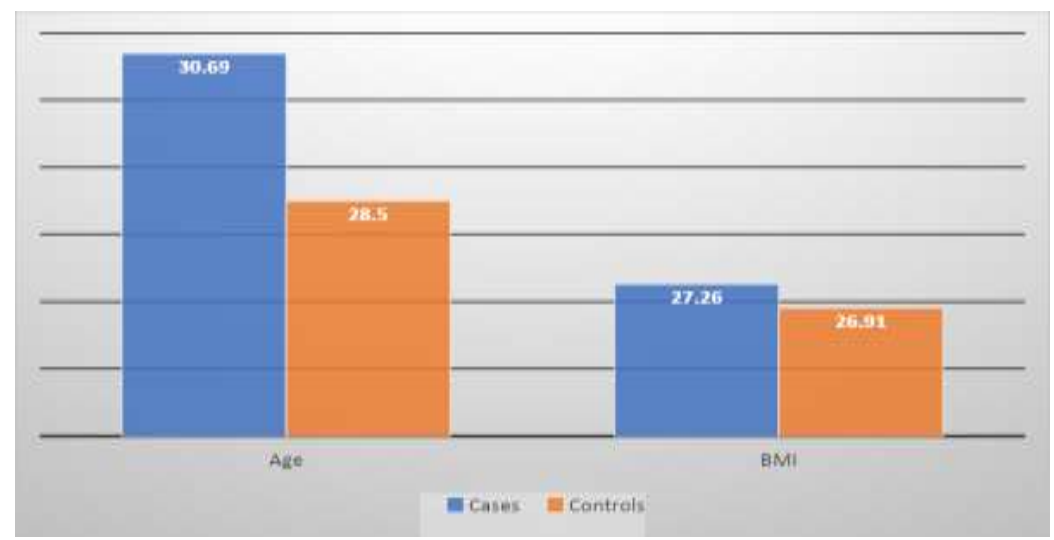

Fig (2) Age and BMI of the two studied groups.

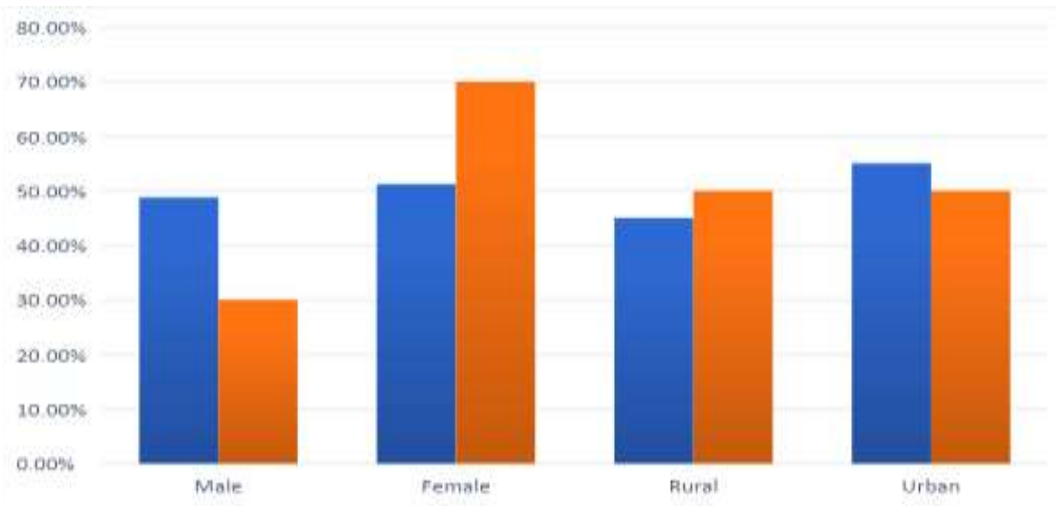

Fig (3) Sex and Residence of the two studied groups.

\section{Discussion}

Psoriasis is a chronic and recurrent skin disease, which has serious negative effects on quality of life (QOL). The prevalence of psoriasis reported in different countries ranges from $0.09 \%$ to $11.43 \%, 2$ but in China, it is $0.47 \%$. Current treatment can only alleviate the symptoms of psoriasis not cure them, leaving a huge burden on family and society. The etiology of the disease is unknown, current research suggests that psoriasis is a complicated disease induced by immune and environmental factors and controlled by interactions of multiple genes. Epidemiological research has confirmed a familial genetic predisposition of psoriasis, with $>20 \%$ of patient having a positive family history. Evidence indicates that psoriasis has a genetic predisposition, with researchers having identified $>80$ susceptibility genes/loci. Gene function studies are under way, which could help us to better understand the pathogenesis of disease and provide new ideas for diagnosis and treatment [11].

The present study included 80 patients 39 (48.8\%) males and $41(51.2 \%)$ females) with psoriasis versus 10 healthy subjects $3(30 \%)$ males and $7(70 \%)$ females) within the same age group (20-40). Family history of psoriasis in Patients group showed that $47(58.8 \%)$ had no previous family history of psoriasis and $33(41.3 \%)$ had previous positive family history of psoriasis while in Control Group $3(30 \%)$ had no previous family history of the disease in question and 7 (70\%) had previous family history of psoriasis. There was no statistically significant differences between the two groups where $\mathrm{P}=0.102$, as assessed via independent $T$ test [12].

Within patients with psoriasis (n80), it was shown that $19(23.8 \%)$ patients had plaque type, $19(23.8 \%)$ of them had guttate type, $14(17.5 \%)$ had inverse type of psoriasis, 14(17.5\%) had pustular type and $14(17.5 \%)$ had erythrodermic type of the disease. Patient's Psoriasis PASI score in Study group ranged between 1545 with a mean value of $29.72 \pm 9.302$. Patients with psoriasis arthropathy were excluded. It has been shown that cutaneous psoriasis [e.g. psoriasis vulgaris (PsV)] and psoriatic arthritis (PsA) are complex heterogeneous diseases. The soluble and cellular mediators of these closely related diseases are being elucidated through genetic approaches such as genome-wide association studies (GWAS), as well as animal and molecular models. Clearly, PsV and PsA are highly variable in terms of their clinical manifestations and this heterogeneity can partially be explained by differences in HLA-associations (HLA-Cw*0602 versus HLA-B*27, for example). In addition, there are numerous other genetic susceptibility loci (LCE3, CARD14, NOS2, NFKBIA, PSMA6, ERAP1, TRAF3IP2, IL12RB2, IL23R, IL12B, TNIP1, TNFAIP3, TYK2) and geoepidemiologic factors that contribute to the wide 
variability seen in psoriasis [13]. The gene study of iNO gene revealed considerable results

On the contrary, it had been reported a statistically significant iNOS gene (substitution rs4795067) polymorphism associated with psoriasis in a genomewide association study (GWAS) [14].

It seems logical to adopt GWAS in studying gene polymorphisms in a rather complex disease such as psoriasis. The GWAS is a strategy to find genetic variations that affect complex traits. It is based on highthroughput genotyping technology, through analysis of millions of single nucleotide polymorphisms to find relevant clinical manifestations or phenotypic traits. GWAS provides a new way to study the genetic characteristics of complex diseases, allowing detection throughout the patient's whole genome to identify mutation allele frequencies rather than selecting diseasecausing genes as candidate genes. Furthermore, it helps to identify genes and pseudoautosomal regions that have not been previously discovered, and thus can provide more clues to the pathogenesis of complex diseases such as psoriasis [11].

\section{References}

[1] A.N. AbdelNoor , N .Al-Akl, Factors Involved in the Pathogenesis of Psoriasis Advanced Studies in Medical Sciences, Vol. 1(2), PP.75 - 94, 2013.

[2] W.B.Kim, D. Jerome, J. Yeung, "Diagnosis and management of psoriasis," Can. Fam. Physician, Vol. 63( 4), PP.278-285, 2017.

[3] M.Wolf, A. Shnyra, Autoimmune Mechanisms of Psoriasis: Pathogenic Role of the IL-23/IL-17 Axis,J .Autoimmune Disorders, Vol. 4 (1),PP.5-11, 2018.

[4] M. Ayala-Fontánez, S. Thomas , S .McCormick , Current knowledge on psoriasis and autoimmune diseases Psoriasis: Targets and Therapy, Vol. 6,PP.32-48, 2016.

[5] J.E. Greb, A.M. Goldminz, J.D. Elder, Psoriasis, Nature Reviews, Vol. 2 (1),PP.33-43, 2016.

[6] N.M. Iverson, E.M. Hoffferber , J.A. Stapleton , Nitric oxide sensors for biological applications , Chemosensors, Vol. 6,PP.8-28,2018.

[7] Y. Zhao, P.M. Zanhoutte, W.S. Leung, Vascular Nitroc Oxide: Beyond eNOS. J Pharmacol SCI, Vol. 129 ,PP.83-94,2015.

[8]U. Forstermann, W.C. Sessa, Nitric oxide synthases: regulation and function. Eur Heart $\mathrm{J}$, Vol. 33(7),PP.829-37, 2012.

[9]W. B. Kim, D. Jerome, J. Yeung, "Diagnosis and management of psoriasis," Can. Fam. Physician, Vol. 63( 4), PP.278-285, 2017.

[10] M. M. Goodman, G. M. White, A. McCormick, J. McCullough, G. Weinstein, "Cyclosporine therapy for psoriasis: a cell cycle-derived dosing schedule," $J$. Am. Acad. Dermatol; Vol. 27( 4), PP.594-598, 1992.

[11] D. Ran, M .Cai, X. Zhang ,Genetics of Psoriasis.recision Clinical Medicine, Vol.2(2), PP.120-130, 2019

[12] H.A. David ,The beginings of randomization tests.Am Stat, Vol.62, PP.70-73, 2008.

[13] B.A. Sukhov, I.E. Adamopoulos, D. Phil ,Interactions of the immune system with skin and bone tissue in psoriatic arthritis: A Comprehensive Review. Clin Rev Allergy Immunol, Vol. 51(1), PP.87-99, 2016.

[14] P.E. Stuart, R.P. Nair, E. Ellinghaus, Genome-wide association analysis identifies three psoriasis susceptibility loci. Nature Genetics, Vol. 42 (11), PP.1000-1004, 2010. 